\title{
On a Characterization of Infinitely Divisible Characteristic Functionals on a Hilbert Space
}

\author{
B. L. S. Prakasa Rao
}

Summary. A characterization of infinitely divisible characteristic functionals on a Hilbert space, analogous to that of Johansen [1], is given.

\section{Introduction}

Let $X$ be a separable Hilbert space, $(x, y)$ denotes its inner product and $\|x\|$ the norm. Let $\mu$ be a probability measure on the $\sigma$-field of Borel subsets of $X$. The characteristic functional $\hat{\mu}(\cdot)$ is defined by

$$
\hat{\mu}(y)=\int e^{i(x, y)} d \mu(x)
$$

for $y \in X$.

Definition 1.1. A positive semi-definite Hermitian operator $A$ on $X$ is called an $S$-operator if it has finite trace. The class of sets $\{x:(S x, x)<t\}$ where $S$ runs over $S$-operators and $t$ over positive numbers forms a neighborhood system at the origin for a certain topology on $X$ which is called the $S$-topology. A net $\left\{x_{\alpha}\right\}$ converges to zero in $S$-topology if and only if $\left(S x_{\alpha}, x_{\alpha}\right)$ converges to zero for every $S$-operator $S$.

We have the following characterization of characteristic functionals on a Hilbert space due to Sazanov [3].

Theorem 1.1. In order that a function $\hat{\mu}(\cdot)$ may be the characteristic functional of a distribution $\mu$ on $X$, it is necessary and sufficient that (i) $\hat{\mu}(0)=1$ (ii) $\hat{\mu}(\cdot)$ be positive definite and (iii) $\hat{\mu}(\cdot)$ be continuous at 0 in the $S$-topology.

Our aim in this paper is to give a similar characterization for infinitely divisible characteristic functionals on a Hilbert space. We shall state a few more definitions and the main theorem in the next section. Proof of the theorem is given in Section 3. For more details on probability measures and characteristic functionals on a Hilbert space, the reader is referred to either Parthasarathy [2], or Varadhan [4].

\section{Infinitely Divisible Distributions}

Definition 2.1. A probability measure $\mu$ on a Hilbert space $X$ is said to be infinitely divisible if for every positive integer $n$,

$$
\mu=\underbrace{\lambda_{n} * \lambda_{n} * \cdots * \lambda_{n}}_{n \text { times }}
$$

where $\lambda_{n}$ is a probability measure on $X$ and $*$ denotes the convolution operation. 
Theorem 2.1. If $\mu$ is an infinitely divisible distribution on $X$ and $\hat{\mu}$ is its characteristic functional, then $\hat{\mu}(y) \neq 0$ for all $y \in X$.

We refer the reader to Parthasarathy [2] for a proof of this theorem. We also note that a Hilbert space has no nontrivial compact subgroups and hence there are no nontrivial idempotent distributions. Further more, if $\hat{\mu}(\cdot)$ is the characteristic functional of an infinitely divisible distribution on $X$, then it has a unique representation of the form $\hat{\mu}(y)=e^{\chi(y)}$ for some complex valued function $\chi(\cdot)$ on $X$ by Theorem 4.10 of Parthasarathy [2]. We shall call $\chi(\cdot)$ the logarithm of the characteristic functional $\hat{\mu}(\cdot)$. The main theorem of the paper will be stated now.

Theorem 2.2. In order that $\chi(\cdot)$ be the logarithm of a characteristic functional of an infinitely divisible distribution $\mu$ on $X$ it is necessary and sufficient that

(i) $\chi(0)=0, \chi(y)=\overline{\chi(-y)}$,

(ii) $\chi(\cdot)$ is continuous at 0 in S-topology, and

(iii) for every choice $y_{i}, 1 \leqq i \leqq N$ in $X$ and complex numbers $\alpha_{i}, 1 \leqq i \leqq N$ such that $\sum_{i=1}^{N} \alpha_{i}=0$,

$$
\sum_{i=1}^{N} \sum_{j=1}^{N} \chi\left(y_{i}-y_{j}\right) \alpha_{i} \bar{\alpha}_{j} \geqq 0 .
$$

Before we give a proof of the above theorem, we shall state a lemma. This lemma has been proved by Johansen [1] for complex valued functions on the real line. Proof of the lemma for complex valued functions on a Hilbert space is exactly similar to the proof given by Johansen [1] and hence it is omitted.

Lemma 2.3. Let $\chi(\cdot)$ be any complex valued function on $X$ such that $\chi(0)=0$, $\chi(y)=\overline{\chi(-y)}$. Then the following conditions are equivalent. $\lambda \geqq 0$,

(i) For every choice $y_{i}, 1 \leqq i \leqq N$ in $X$ and complex numbers $\alpha_{i}, 1 \leqq i \leqq N$ and

$$
\sum_{i=1}^{N} \sum_{j=1}^{N} e^{\lambda \chi\left(y_{i}-y_{j}\right)} \alpha_{i} \bar{\alpha}_{j} \geqq 0 .
$$
that

(ii) For every choice $y_{i}, 1 \leqq i \leqq N$ in $X$ and complex numbers $\alpha_{i}, 1 \leqq i \leqq N$ such

$$
\sum_{i=1}^{N} \alpha_{i}=0, \quad \sum_{i=1}^{N} \sum_{j=1}^{N} \chi\left(y_{i}-y_{j}\right) \alpha_{i} \bar{\alpha}_{j} \geqq 0 .
$$

(iii) For every choice $y_{i}, 1 \leqq i \leqq N$ in $X$ and complex numbers $\alpha_{i}, 1 \leqq i \leqq N$,

$$
\sum_{i=1}^{N} \sum_{j=1}^{N}\left[\chi\left(y_{i}-y_{j}\right)-\chi\left(y_{i}\right)-\chi\left(-y_{j}\right)\right] \alpha_{i} \bar{\alpha}_{j} \geqq 0 \text {. }
$$

\section{Proof of Theorem 2.2}

Necessity. Suppose $\hat{\mu}(\cdot)$ is the characteristic functional of an infinitely divisible distribution $\mu$ on $X$. Let $\chi(\cdot)$ be the logarithm of $\hat{\mu}(\cdot)$. (i) follows from the definition of a characteristic functional. (ii) follows from Theorem 1.1. Since $\hat{\mu}$ is an infinitely divisible characteristic functional $\hat{\mu}^{1 / n}$ is a uniquely determined characteristic 
functional for every $n$ and hence $\hat{\mu}^{r}$ is a characteristic functional for every rational number $r$. Hence by Theorem 1.1,

$$
\sum_{i=1}^{N} \sum_{j=1}^{N}\left[\hat{\mu}\left(y_{i}-y_{j}\right)\right]^{r} \alpha_{i} \bar{\alpha}_{j} \geqq 0
$$

for every choice of $y_{i}, 1 \leqq i \leqq N$ in $X$ and complex numbers $\alpha_{i}, 1 \leqq i \leqq N$. Let $r_{n}$ be any sequence of rational numbers approaching $\lambda \geqq 0$ as $n$ approaches $\infty$. By taking limits as $n \rightarrow \infty$ on both sides of (3.1), we get that

$$
\sum_{i=1}^{N} \sum_{j=1}^{N}\left[\hat{\mu}\left(y_{i}-y_{j}\right)\right]^{\lambda} \alpha_{i} \bar{\alpha}_{j} \geqq 0
$$

for every choice of $y_{i}, 1 \leqq i \leqq N$ and complex numbers $\alpha_{i}, 1 \leqq i \leqq N$, and $\lambda \geqq 0$. This proves (iii) in view of Lemma 2.3 since $\hat{\mu}(\cdot)=e^{x(\cdot)}$. This completes the proof of the necessity of the conditions (i), (ii) and (iii) of the theorem.

Sufficiency. Suppose $\chi(\cdot)$ is a complex valued functional which satisfies (i), (ii) and (iii) of the theorem. Define $\psi(y)=e^{\chi(y)}$ for any $y \in X$. Clearly $[\psi(0)]^{\lambda}=1$ for any $\lambda \geqq 0$ and $\psi^{\lambda}$ is continuous at 0 in $S$-topology for any $\lambda \geqq 0$. Since

$$
\sum_{i=1}^{N} \sum_{j=1}^{N} \chi\left(y_{i}-y_{j}\right) \alpha_{i} \bar{\alpha}_{j} \geqq 0
$$

for every choice of $y_{i}, 1 \leqq i \leqq N$ in $X$ and complex numbers $\alpha_{i}, 1 \leqq i \leqq N$ such that $\sum_{i=1}^{N} \alpha_{i}=0$, Lemma 2.3 implies that

equivalently

$$
\sum_{i=1}^{N} \sum_{j=1}^{N} e^{\lambda \chi\left(y_{i}-y_{j}\right)} \alpha_{i} \bar{\alpha}_{j} \geqq 0
$$

$$
\sum_{i=1}^{N} \sum_{j=1}^{N}\left[\psi\left(y_{i}-y_{j}\right)\right]^{\lambda} \alpha_{i} \bar{\alpha}_{j} \geqq 0
$$

for every choice of $y_{i}, 1 \leqq i \leqq N$ in $X$ and every choice of complex numbers $\alpha_{i}$, $1 \leqq i \leqq N$. In other words $\psi^{\lambda}$ is positive definite for every $\lambda \geqq 0$. This fact together with earlier remarks prove that $\psi^{\lambda}$ is a characteristic functional for every $\lambda \geqq 0$ by Theorem 1.1. Hence $\psi$ is an infinitely divisible characteristic functional which proves the sufficiency of the conditions (i), (ii) and (iii) of the theorem.

\section{References}

1. Johansen, S.: An application of extreme point methods to the representation of infinitely divisible distributions. Z. Wahrscheinlichkeitstheorie verw. Geb. 5, 304-316 (1966).

2. Parthasarathy, K. R.: Probability measures on metric spaces. New York: Academic Press 1967.

3. Sazanov, V.: A remark on characteristic functionals. Theor. Probab. Appl. 3, $188-192$ (1958).

4. Varadhan, S. R. S.: Limit theorems for sums of independent-random variables with values in a Hilbert space. Sankhya, Ser. A 24, 213-238 (1962).

Dr. B. L. S. Prakasa Rao

Department of Mathematics Indian Institute of Technology Kanpur, U. P./India 